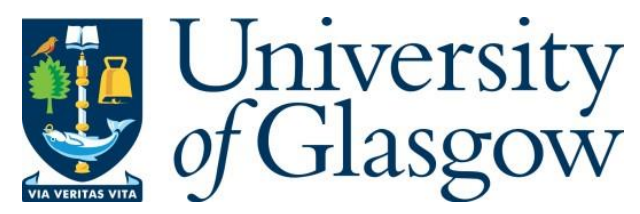

Liao, X., Jiang, T., Huang, Z., and Cochran, S. (2016) Loss Characterisation of Piezocrystals Under Elevated Environmental Conditions. In: 2016 Joint IEEE

International Symposium on the Applications of Ferroelectrics, European Conference on Application of Polar Dielectrics, and Piezoelectric Force Microscopy Workshop (ISAF/ECAPD/PFM), Darmstadt, Germany, 21-25 Aug 2016, ISBN 9781509018710.

There may be differences between this version and the published version. You are advised to consult the publisher's version if you wish to cite from it.

http://eprints.gla.ac.uk/136415/

Deposited on: 13 February 2017

Enlighten - Research publications by members of the University of Glasgow http://eprints.gla.ac.uk 


\title{
Loss Characterisation of Piezocrystals under Elevated Environmental Conditions
}

\author{
Xiaochun Liao' ${ }^{1}$, Tingyi Jiang ${ }^{2}$, Zhihong Huang 2 , Sandy Cochran ${ }^{1}$, \\ ${ }^{1}$ School of Engineering, University of Glasgow, Glasgow, G12 8QQ, UK \\ ${ }^{2}$ School of Science and Engineering, University of Dundee, Dundee, DD1 4HN, UK
}

\begin{abstract}
Relaxor-based piezoelectric single crystals have experienced three generations of development, from binary (e.g. PMN-PT) through ternary (e.g. PIN-PMN-PT) to doped ternary (e.g. Mn:PIN-PMN-PT). With improved composition and other relevant factors, these materials exhibit an extraordinary degree of piezoelectricity and ultrahigh electromechanical coupling coefficients, making them suitable for applications requiring high sensitivity and high bandwidth. With further increases in rhombohedral-to-tetragonal phase transition temperature $\left(T_{R T}\right)$, coercive field $\left(E_{C}\right)$ and mechanical quality factor $\left(Q_{m}\right)$, these piezocrystals can now be expected to work at elevated temperature, $T$, and pressure, $P$, and with high electric field drive. However, in operation, material properties can vary and performance can degrade significantly because of these elevated conditions, and the situation can be exacerbated by losses in the materials, necessitating proper characterisation of loss factors. In this paper, we report an investigation of three different loss characterisation methods then propose one combined method, demonstrating its use on TE-mode plates of PIN-PMN-PT and Mn:PIN-PMN-PT. Characterisation was performed using impedance spectroscopy for $20^{\circ} \mathrm{C} \leqslant T \leqslant 100^{\circ} \mathrm{C}$ and $0 \mathrm{MPa} \leqslant$ $P \leqslant 60$ MPa. Results relating to dielectric, elastic and piezoelectric losses are reported, with detailed analysis and comparisons.
\end{abstract}

Keywords- loss; loss characterisation; quality factor; dielectric; elastic; piezoelectric; ferroelectrics; temperature; pressure

\section{INTRODUCTION}

Relaxor-based piezoelectric single crystals ("piezocrystals") have now developed through three generations, exemplified by binary PMN-PT (Gen. I), ternary PIN-PMN-PT (Gen. II) and doped ternary Mn:PIN-PMN-PT (Gen. III), motivated by improved piezoelectric performance compared with piezoceramics. These piezocrystals exhibit extraordinary piezoelectric properties, e.g. $d_{33}=1500 \mathrm{pC} / \mathrm{N}$ and $d_{15}=2190 \mathrm{pC} / \mathrm{N}$, compared with $d_{33} \approx 300 \mathrm{pC} / \mathrm{N}$ for PZT4. This makes them suitable for a wide range of passive and active applications requiring high bandwidth and high sensitivity piezoelectric transduction [1].

The three generations of piezocrystal have been further improved by modifying their material systems to provide higher coercive field $\left(E_{C}\right)$, mechanical quality factor $\left(Q_{m}\right)$ and rhombohedral-to-tetragonal phase transition temperature $\left(T_{R T}\right)$. These changes are beneficial in expanding the boundaries of use under elevated conditions where high environmental temperature, $T$, pressure, $P$, and active electric field drive are needed [2]. However, significant material property variations caused by elevated external conditions have been reported $[3,4]$, demonstrating the need to evaluate piezocrystal stability and reliability in performance in conditions matching practical scenarios.

One essential aspect of evaluation, besides basic material properties, is measurement of loss factors which strongly relate to both stability with temperature increases caused by high drive conditions and performance expressed by figures of merit (FOMs). One example is $k_{i j}^{2} Q_{m}$ for active underwater transducers, where $k_{i j}$ is the relevant electromechanical coupling coefficient, and another is $d_{h} g_{h} / \tan \delta_{e}$ for passive underwater hydrophones, where $d_{h}$ and $g_{h}$ are the piezoelectric constants under hydrostatic conditions.

Losses in piezoelectric material comprise three parts: elastic loss, dielectric loss and piezoelectric loss, all generating heat, lowering energy efficiency and degrading performance through material property variation. There are three basic methods to characterise the loss factors:

(1) observation of hysteresis loops relating four variables, electric displacement $D$, electric field $E$, mechanical strain $S$, and mechanical stress $T$ [5];

(2) derivation from material properties using the ratios of the real and imaginary properties; and

(3) equation-based derivation for each loss factor [6].

However, the three methods do not always agree. Additionally, loss factor measured has previously been conducted mainly for piezoceramics. It is therefore important to investigate loss characterization methods for piezocrystals both at and beyond ambient conditions.

In the study described here, aiming at characterising the loss factors of piezocrystals under elevated environmental conditions, we first examine the three different loss characterisation methods and proposed the adopted method for each loss factor in Section II. Then, in Section III, experimental arrangements to apply elevated temperature and pressure for PIN-PMN-PT and Mn:PIN-PMN-PT are explained, with measurements based on electric impedance magnitude spectroscopy. Detailed loss characterization results and discussions are presented for elastic, dielectric, and piezoelectric losses in Section IV. Finally, conclusions and suggestions for future work are presented in Section V.

\section{LOSS CHARACTERISATION METHODS}

Three types of loss mechanisms degrade the functional performance of piezoelectric materials in operation: elastic 
loss, dielectric loss, and piezoelectric loss. Elastic, or mechanical loss, relates to the intrinsic damping or "internal friction" of the material, expressed by a loss tangent, $\tan \phi$, or, in practical situations, by a mechanical quality factor, $Q_{m}$, relating to a time constant, $\tau$, for attenuation of vibration. Dielectric loss represents the inherent dissipation of electromagnetic energy in a dielectric material when an alternating voltage/current is applied, with a ceramic capacitor as the prime example. The energy is mainly converted into heat and the dielectric loss can be expressed in terms of a loss tangent $\tan \delta$. To date, there has been no clear agreement on the origin of the piezoelectric losses. However, it is generally thought to be linked with the dissipation in energy conversion from mechanical to electrical or from electrical to mechanical, energy, again expressed by a loss tangent, $\tan \theta$.

Mathematically, these loss tangents can be expressed as complex numbers for piezoelectric materials. Combining the field variables, the definitions are given by:

$$
\begin{aligned}
\varepsilon^{T *} & =\varepsilon^{T}\left(1-j \tan \delta^{\prime}\right) \\
s^{E *} & =s^{E}\left(1-j \tan \emptyset^{\prime}\right) \\
d^{*} & =d\left(1-j \tan \theta^{\prime}\right) \\
\beta^{S *} & =\beta^{S}(1+j \tan \delta) \\
c^{D *} & =c^{D}(1+j \tan \emptyset) \\
h^{*} & =h(1+j \tan \theta)
\end{aligned}
$$

In these equations, $j$ is the imaginary unit and the asterisk indicates a complex number. $\varepsilon^{T}$ is the dielectric permittivity under constant stress, $T . s^{E}$ is the elastic compliance under constant electric field, $E . d$ is the piezoelectric constant. $\beta^{S}$ is the inverse dielectric constant under constant strain, $S . c^{D}$ is the elastic stiffness under constant electric displacement, $D . h$ is the inverse piezoelectric constant. $\tan \delta^{\prime}, \tan \phi^{\prime}$ and $\tan \theta^{\prime}$ represent the intensive dielectric, elastic and piezoelectric loss factors repectively, under stress free and short circuit conditions. Without prime notation, $\tan \delta$, $\tan \phi$ and $\tan \theta$ represent the extensive loss factors, associated with clamped and open circuit conditions [6].

\section{A. Hysteresis method}

The three types of losses are associated with hysteresis responses between four relationships: (1) strain versus stress for elastic loss; (2) electric displacement versus electric field for dielectric loss; and (3) strain versus electric field or (4) electric displacement versus stress for piezoelectric loss. From the perspective of energy, the area beneath the response curve represents the energy storage, and the area within the hysteresis loop represents the energy dissipation relating to loss.

Taking the hysteretic response for dielectric loss as an example, as shown in Fig.1, the stored energy, $U_{E}$, with $E$ increasing from 0 to $E_{0}$, and the energy dissipation, $w_{E}$, within the whole hysteresis cycle, are given by:

$$
\begin{gathered}
U_{E}=\frac{1}{2} \varepsilon E_{0}^{2} \\
w_{E}=\pi \varepsilon E_{0}^{2} \tan \delta^{\prime}
\end{gathered}
$$

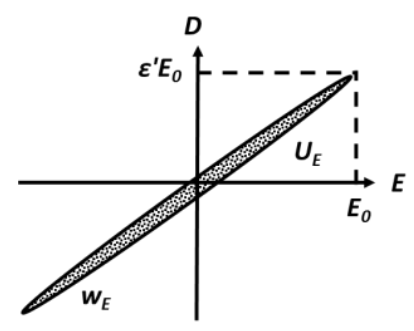

Figure 1. Hysteresis reponse of $D$ versus $E$ for dielectric loss, $\tan \delta$

By combining Eqns. 7 and 8, the free dielectric loss can be obtained as:

$$
\tan \delta^{\prime}=\frac{w_{E}}{2 \pi U_{E}}
$$

This method is directly based on the conception of energy, and involves the use of a Sawyer-Tower circuit to make measurements.

In the related experiments, specific mechanical arrangements are needed to control the strain and stress response for the characterisation of elastic and piezoelectric losses; these are difficult and always invasive of the piezoelectric samples.

\section{B. Material property based method}

Considering that all the loss factors can be expressed mathematically as complex numbers, another feasible characterisation method is to derive the loss factors from the ratio of the imaginary and real parts of a specific material property, $M P$, simply as:

$$
\text { loss factor }=\operatorname{imag}(M P) / \operatorname{real}(M P)
$$

For dielectric loss, $\tan \delta$ ' and $\tan \delta$ can be derived from the complex forms of free and clamped dielectric permittivity respectively, with a straightforward measurement using an LCR meter or an impedance analyser (e.g., 4395A, Agilent/Keysight, Santa Clara, CA, USA). For elastic and piezoelectric losses, the complex forms of elastic and piezoelectric properties can be obtained by fitting them to impedance spectroscopy curves measured with a calibrated electrical impedance analyser. Commercial software is available for the fitting process (PRAP 2.2, TASI Technical Software Inc., Ontario, Canada). The accuracy of this method depends on the fitting algorithm for the piezoelectric material under analysis, and requires clear resonant and anti-resonant features to be obtained by measurement.

\section{Equation based method}

According to the IEEE standard [7], some specific loss factors are expressed in equations for simple derivation. For elastic loss, $\tan \phi$, relating to the mechanical quality factor, $Q_{m}$, they are given as:

$$
\begin{gathered}
\tan \emptyset=1 / Q_{m} \\
Q_{m}=f_{0} / \Delta f
\end{gathered}
$$

where $f_{0}$ is the central frequency of the peak response and $\Delta f$ is the $-3 \mathrm{~dB}$ bandwidth. 
Loss factors for specific vibration modes have also been systematically reported in previous research [6] and are here considered to be useful to investigate the loss in piezocrystals. Taking the TE mode as an example, losses are expressed as:

$$
Q_{a}=1 / \tan \emptyset_{33}
$$

$\frac{1}{Q_{r}}=\frac{1}{Q_{a}}+\frac{2}{k_{t}^{2}-1+\Omega_{A}^{2} / k_{t}^{2}}\left(\tan \delta_{33}+\tan \emptyset_{33}-2 \tan \theta_{33}\right)$

where $Q_{r}$ and $Q_{a}$ are the mechanical quality factors for the resonant and anti-resonant frequencies, $k_{t}$ is the electromechanical coupling coefficient for the TE-mode sample, and $\Omega_{A}$ is given by:

$$
\Omega_{A}=\frac{w_{A} b}{2} \sqrt{\frac{\rho}{c_{33}^{D}}}
$$

where $w_{A}$ is the angular frequency at resonance, $b$ is the sample thickness, $\rho$ is the sample density, and $c_{33}{ }^{D}$ is the elastic stiffness constant.

These equations are obtained based on restriction of certain vibrational modes and also mathematical approximations in their derivation. Special attention is required when it is not possible to conform to the restrictions or if the assumed approximation is not valid.

\section{Combined method}

After evaluating the advantages and disadvantages of the three methods outlined above, a combined method is proposed to further examine their applicability, based on measurements with two $5 \times 5 \times 0.5 \mathrm{~mm}^{3}$ TE-mode plates of PIN-PMN-PT and Mn:PIN-PMN-PT.

For dielectric loss, it is proposed to determine the loss factors for free and clamped permittivity, respectively, as:

$$
\begin{aligned}
& \tan \delta_{33}^{\prime}=\operatorname{imag}\left(\varepsilon_{33}^{T}\right) / \operatorname{real}\left(\varepsilon_{33}^{T}\right) \\
& \tan \delta_{33}=\operatorname{imag}\left(\varepsilon_{33}^{S}\right) / \operatorname{real}\left(\varepsilon_{33}^{S}\right)
\end{aligned}
$$

The mechanical quality factors are determined for resonance and anti-resonance, respectively, as:

$$
\begin{aligned}
& Q_{r}=f_{r} / \Delta f_{r} \\
& Q_{a}=f_{a} / \Delta f_{a}
\end{aligned}
$$

The piezoelectric loss is obtained with two methods for comparison, with one based on material properties:

$$
\tan \theta_{33}=\operatorname{imag}\left(h_{33}^{*}\right) / \operatorname{real}\left(h_{33}^{*}\right)
$$

and the other one based on a more complicated equation:

$\tan \theta_{33}=\frac{1}{2}\left(\tan \delta_{33}+\frac{1}{Q_{a}}\right)+\frac{1}{4}\left(\frac{1}{Q_{a}}-\frac{1}{Q_{r}}\right)\left(k_{t}^{2}-1+\frac{\Omega_{A}^{2}}{k_{t}^{2}}\right)$

\section{TEST ARRANGEMENTS}

To assess the effects of environmental temperature and pressure rise on the three types of loss factors, impedance spectra of the piezocrystal samples were measured, with temperature controlled by a fan-assisted oven and uniaxial pressure applied with a material testing machine (MTS, SSID 5966, Instron Ltd., High Wycombe, UK). The temperature started at $20^{\circ} \mathrm{C}$, with the pressure increasing from 0 to $60 \mathrm{MPa}$ Then, the temperature was elevated to $40^{\circ} \mathrm{C}$ with the pressure cycled again $0-60 \mathrm{MPa}$, until the ultimate temperature $100{ }^{\circ} \mathrm{C}$ and pressure $60 \mathrm{MPa}$ were reached, as shown in Fig.2 (a).

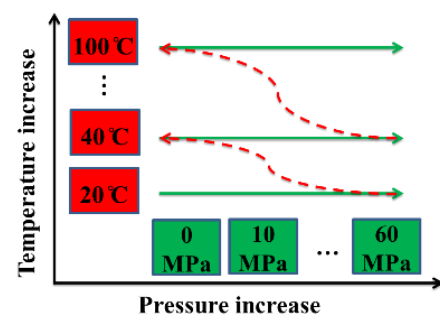

(a)

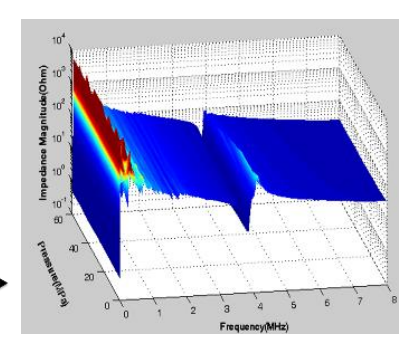

(b)
Figure 2. (a) Test arrangement with externally applied environmental conditions of $20^{\circ} \mathrm{C} \leqslant \mathrm{T} \leqslant 100^{\circ} \mathrm{C}, 0 \mathrm{MPa} \leqslant \mathrm{P} \leqslant 60 \mathrm{MPa}$ and

(b) example of variation of impedance magnitude at $80^{\circ} \mathrm{C}$ under different pressure levels for Mn:PIN-PMN-PT TE-mode sample

The impedance magnitudes of the test samples were obtained, as shown in Fig. 2(b), and the combined method was used to extract the loss factors expressed in Eqns. 16 - 21.

\section{CHARACTERISATION OF LOSS FACTORS}

The free and clamped dielectric losses $\left(\tan \delta_{33}\right.$ ' and $\left.\tan \delta_{33}\right)$ were extracted from the impedance magnitude measurements using Eqns. 16 and 17, as shown in Fig. 3, for both PIN-PMNPT and Mn:PIN-PMN-PT. tan $\delta_{33}$ increased sharply after $80^{\circ} \mathrm{C}$ and tended to stabilise with elevated pressure at $80^{\circ} \mathrm{C}$ and $100^{\circ} \mathrm{C}$. For $\tan \delta_{33}$, a sharp increase was found only for PIN-PMN-PT at $100^{\circ} \mathrm{C}$, and the stabilisation effect with elevated pressure level can be easily seen for Mn:PIN-PMN$\mathrm{PT}$. Both tan $\delta_{33}$ and tan $\delta_{33}$ generally increased with temperature for both materials.
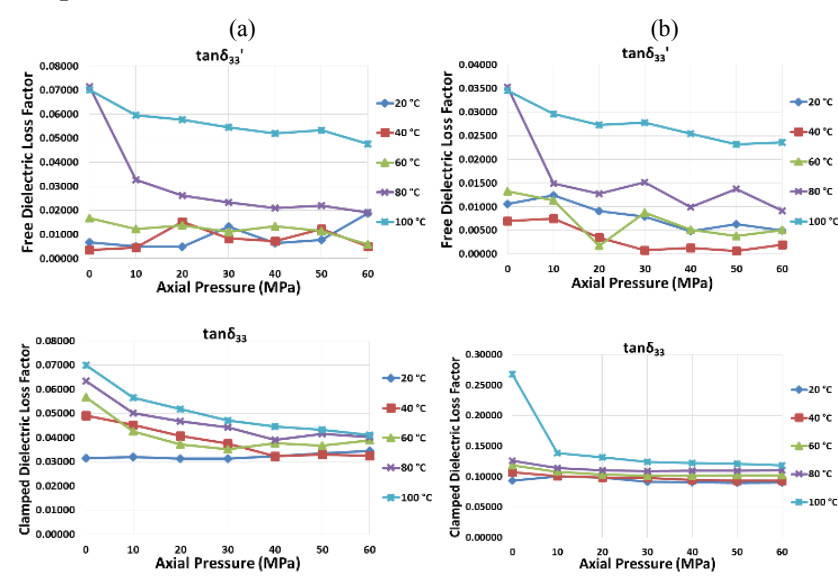
Figure 3. Variations of dielectric loss for (a) Mn:PIN-PMN-PT and (b) PIN-PMN-PT

The mechanical quality factors at resonance and antiresonance, $Q_{r}$ and $Q_{a}$, respectively, for the TE-mode samples were analysed by measured impedance spectra and analysing the results using Eqns. 18 and 19, as shown in Fig 4. $Q_{r}$ decreased sharply as the temperature and pressure rose. For $Q_{a}$, the most significant degradation happened at $20^{\circ} \mathrm{C}$ and it recovered significantly after the pressure was released from $60 \mathrm{MPa}$ back to $0 \mathrm{MPa}$. Both $Q_{r}$ and $Q_{a}$ exhibited recovery in the range 20 - $40 \mathrm{MPa}$ during pressure application.

(a)

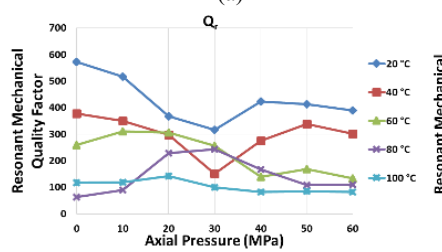

$a_{0}$

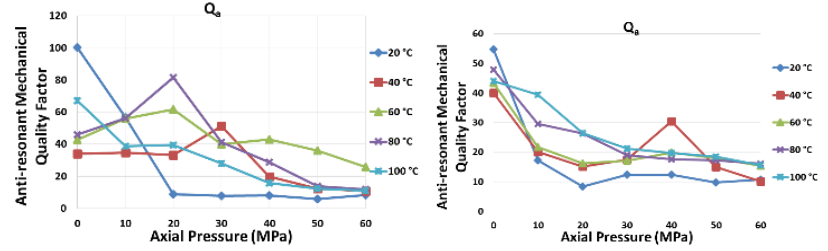

Figure 4. Variations of mechanical quality factor for (a) Mn:PIN-PMN-PT and (b) PIN-PMN-PT

Based on the complex material property, $h_{33}{ }^{*}$, derived by fitting (PRAP 2.2, TASI Technical Software Inc., Ontario, Canada), the piezoelectric loss factor $\tan \theta_{33}$ can be obtained using Eqn. 20, as shown in Fig. 5. For $\tan \theta_{33}$, it increased significantly with elevated pressure to values of 0.45 and 0.3 respectively for Mn:PIN-PMN-PT and PIN-PMN-PT; these values are much higher than the previously expected value of about 0.1 [8].
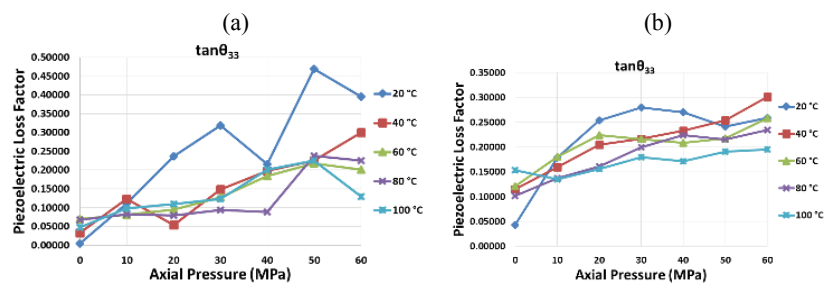

Figure 5. Variations of piezoelectric loss for (a) Mn:PIN-PMN-PT and (b) PIN-PMN-PT, based on complex representations of material properties

Based on the method related to Eqn. 21, the piezoelectric loss factor $\tan \theta_{33}$ was obtained as shown in Fig. 6 . The values followed the same trends as in Fig. 5, but they were extremely high, with some exceeding unity. This may be caused by violation of the approximations presumed in the derivation of the underlying equations. (a)

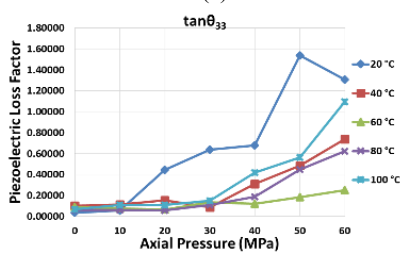

(b)

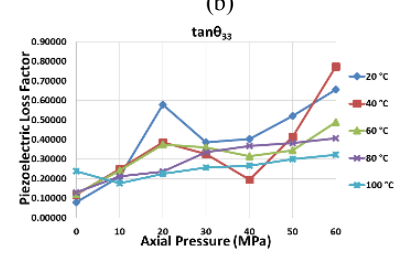

Figure 6. Variations of piezoelectric loss for (a) Mn:PIN-PMN-PT and (b) PIN-PMN-PT, based on Eqn.21

\section{CONCLUSIONS AND FUTURE WORK}

This paper has examined three different methods to characterise the loss factors of piezoelectric materials, and proposed one combined method for use on TE-mode piezocrystal samples of Mn:PIN-PMN-PT and PIN-PMN-PT.

Characterisation results demonstrated significant increases in elastic, dielectric and piezoelectric losses, higher than previous expectation and carrying the potential for severe degradation in functional performance. The results for piezoelectric loss indicated invalidity, most likely because of violation of assumptions in the derivations of the relevant equations. Future work will explore how to characterise three loss factors under high electric field drive for piezocrystals.

\section{ACKNOWLEDGMENTS}

The authors thank UK EPSRC for funding support under project EP/K020013.

\section{REFERENCES}

[1] S. Zhang, et al., "Advantages and challenges of relaxor-PbTiO3 ferroelectric crystals for electroacoustic transducers - A review," Progress in Materials Science, vol. 68, pp. 1-66, 2015.

[2] S. Zhang and F. Li, "High performance ferroelectric relaxor$\mathrm{PbTiO} 3$ single crystals: Status and perspective," Journal of Applied Physics, vol. 111, p. 031301, 2012.

[3] L. Xiaochun, et al., "Functional characterization of piezocrystals monitored under high power driving conditions," in Ultrasonics Symposium (IUS), 2015 IEEE International, 2015, pp. 1-4.

[4] X. Liao, et al., "Functional Piezocrystal Characterisation under Varying Conditions," Materials, vol. 8, p. 5456, 2015.

[5] K. Uchino and S. Hirose, "Loss mechanisms in piezoelectrics: how to measure different losses separately," IEEE Transactions on Ultrasonics, Ferroelectrics, and Frequency Control, vol. 48, pp. 307-321, 2001.

[6] Y. Zhuang, et al., "Methodology for Characterizing Loss Factors of Piezoelectric Ceramics," Ferroelectrics, vol. 470, pp. 260-271, 2014/10/03 2014.

[7] "IEEE Standard on Piezoelectricity," ANSI/IEEE Std 176-1987, p. 01,1988

[8] Z. Yuan, et al., "Derivation of Piezoelectric Losses from Admittance Spectra," Japanese Journal of Applied Physics, vol. 48, p. 041401, 2009. 